\title{
Antibodies against Pneumococcal Capsular Polysaccharides and Natural Anti-Galactosyl (Alpha-Gal) in Patients with Humoral Immunodeficiencies
}

\author{
P. Kralickova, ${ }^{1}$ J. Kuhnova, ${ }^{2}$ O. Soucek, ${ }^{1}$ P. Vodarek, ${ }^{3}$ P. Zak, ${ }^{3}$ M. Simkovic, ${ }^{3}$ M. Motyckova, ${ }^{3}$ \\ L. Smolej, ${ }^{3}$ E. Mala, ${ }^{1}$ C. Andrys, ${ }^{1}$ J. Krejsek, ${ }^{1}$ and V. Thon ${ }^{4,5}$ \\ ${ }^{1}$ Institute of Clinical Immunology and Allergology, Faculty of Medicine in Hradec Kralove, Charles University and University \\ Hospital Hradec Kralove, Hradec Kralove, Czech Republic \\ ${ }^{2}$ Department of Mathematics, Faculty of Science, University of Hradec Kralove, Hradec Kralove, Czech Republic \\ ${ }^{3} 4$ th Department of Internal Medicine-Haematology, Faculty of Medicine, Charles University and University Hospital Hradec \\ Kralove, Hradec Kralove, Czech Republic \\ ${ }^{4}$ Department of Clinical Immunology and Allergy, Faculty of Medicine, Masaryk University, St. Anne's University Hospital, Brno, \\ Czech Republic \\ ${ }^{5}$ RECETOX, Faculty of Science, Masaryk University, Brno, Czech Republic
}

Correspondence should be addressed to P. Kralickova; pavlina.kralickova@fnhk.cz

Received 18 April 2017; Revised 27 July 2017; Accepted 7 September 2017; Published 17 December 2017

Academic Editor: Senthami R. Selvan

Copyright (C) 2017 P. Kralickova et al. This is an open access article distributed under the Creative Commons Attribution License, which permits unrestricted use, distribution, and reproduction in any medium, provided the original work is properly cited.

\begin{abstract}
Humoral deficiencies represent a broad group of disorders. The aim of the study was to compare the levels of antibodies against pneumococcal capsular polysaccharides (anti-PCP) and natural anti-galactosyl (anti-Gal) antibodies in (1) patients with chronic lymphocytic leukaemia (CLL), (2) patients with common variable immunodeficiency (CVID), and (3) a healthy population and to explore their diagnostic and prognostic potential. Serum immunoglobulin levels and levels of anti-Gal IgG, IgA, and IgM and anti-PCP IgG and IgG2 were determined in 59 CLL patients, 30 CVID patients, and 67 healthy controls. Levels of IgG, IgA, IgM, anti-Gal IgA, anti-Gal IgM, and anti-PCP IgA were lower in CLL and CVID patients than in healthy controls ( $p$ value for all parameters $<0.0001)$. Decrease in the levels of IgA, IgM, anti-Gal IgA, and anti-PCP IgA was less pronounced in the CLL group than in the CVID group. IgA decline, anti-Gal IgA, anti-PCP IgA, and anti-PCP IgG2 were negatively correlated with CLL stage. We devise the evaluation of anti-Gal antibodies to be a routine test in humoral immunodeficiency diagnostics, even in cases of immunoglobulin substitution therapy. Significant reductions, mainly in anti-Gal IgA, IgM, and anti-PCP IgA levels, may have prognostic importance in CLL patients.
\end{abstract}

\section{Introduction}

B cell lymphocytic leukaemia (CLL) is the most common leukaemia in western European adults [1]. CLL is particularly frequent in the elderly population, with an average age at diagnosis of 72 years. The disease course and survival time are widely variable $[1,2]$. CLL is characterized by an accumulation of clonal lymphocytes with a specific immunophenotype $\left(\mathrm{CD}^{+}, \mathrm{CD}^{+} 9^{+}\right)$in the bone marrow, peripheral blood, and secondary lymphoid organs and leads to organomegaly and suppression of physiological haematopoiesis [2]. Another clinical feature of CLL is complex alterations of the immune system, leading to higher susceptibility to infections, higher incidence of secondary malignancies, and autoimmune phenomena, such as autoimmune haemolytic anaemia and immune thrombocytopaenia $[3,4]$. Infectious complications are the major cause of morbidity and mortality in more than $50 \%$ of all 
CCL-related deaths $[5,6]$. The most common immune system defect in CLL patients is hypogammaglobulinaemia. Its severity correlates with the duration and stage of disease and is observed even in patients who have never been treated for CLL [7].

The main consequence of hypogammaglobulinaemia is increased frequency of respiratory tract infections caused by encapsulated bacteria (Streptococcus pneumoniae, Haemophilus influenzae, and Staphylococcus aureus) [8]. CLL patients with antibody failure must be identified based on immunization and monitoring, and patients who are clinically symptomatic should be protected by administration of prophylactic antibiotics and/or immunoglobulin substitution therapy $[5,9-11]$.

Common variable immunodeficiency (CVID) is a disease (or likely group of diseases) caused by a deficiency in primary antibody production with enormous heterogeneity in clinical presentation. The patients are characterised by decreased IgG levels, accompanied by decreased IgA and/or IgM levels, and highly disturbed specific antibody responses to antigen challenge. Other types of hypogammaglobulinaemia must be distinguished from this type [12]. Recurrent bacterial infections of the respiratory and gastrointestinal tracts are common symptoms. A significant proportion of patients also exhibit different features of immune dysregulation, including autoimmune diseases, lung granulomatous/interstitial inflammation, enteropathy, and malignancy $[13,14]$. Adequate long-life immunoglobulin substitution therapy is indicated for all established CVID patients.

Evaluation of antibody production capacity is important in humoral immunodeficiency diagnostics and is a key criterion for the indication of immunoglobulin substitution therapy. Patients with hypogammaglobulinaemia should be examined for responses to T-dependent and T-independent antigens $[11,12]$. Evaluation of natural antibodies may also be important.

Anti-galactosyl (anti-Gal) antibodies are the most abundant natural antibodies in humans and are naturally produced in apes and Old World monkeys. The ligand of anti-Gal is a carbohydrate antigen with the structure anti-Gal: $\alpha 1-3 \mathrm{Gal} \beta 1-4 \mathrm{GlcNAc-R}$ [15]. These antibodies can be detected in IgG, IgA, IgM, and IgE. Anti-Gal IgE is produced in some individuals and causes allergies to red meat, bovine gelatin, and cetuximab [16, 17]. Anti-Gal IgM and IgG also mediate rejection of xenografts expressing the $\alpha$-gal epitope [15].

There is no detectable anti-Gal IgM and IgA in the cord blood of newborns, whereas anti-Gal IgG is present at similar levels in both the neonate and mother because of transplacental transfer during pregnancy. These antibodies are subsequently replaced by anti-Gal antibodies produced by the neonate, and their levels increase significantly during the first two years of life [18]. The plasma concentrations remain mostly stable, with some interindividual variability throughout the patient's life. Bernth-Jensen et al. found lower plasma levels of these antibodies in subjects with blood group B [19].

The aim of the study was to evaluate the diagnostic and prognostic role of antibodies against pneumococcal capsular polysaccharide (anti-PCP) and natural anti-Gal antibodies in
CLL patients with secondary antibody deficiencies and patients with CVID.

\section{Material and Methods}

2.1. Study Design. The study protocol was approved by the local ethics committee. Participants in this study comprised a Czech population (Caucasian), including 59 patients with CLL (age range, 34-88 years, mean age, $66 \pm 10$ years; 38 men, 21 women), 30 CVID patients (age range, 18-82 years, mean age, $46 \pm 16$ years; 12 men, 18 women), and 67 sex- and age-matched healthy individuals (age range, 20-86 years, mean age, $56 \pm 17$ years; 37 men, 30 women).

The CLL patients did not receive any immunoglobulin replacement therapy or chemotherapy, anti-CD-20, or steroid therapy. Rai stage at the time of blood sample collection was $0,1,2,3$, and 4 in $14,8,14,15$, and 8 patients, respectively. Other characteristics of the CLL cohort are summarized in Table 1. All CVID patients fulfilled the criteria of the Pan-American Group for Immunodeficiency and European Society for Immunodeficiencies [20]. They were regularly administered intravenous $(n=21)$ or subcutaneous $(n=9)$ immunoglobulins at monthly doses of $368 \pm 149 \mathrm{mg} /$ $\mathrm{kg}$ (range, $170-940 \mathrm{mg} / \mathrm{kg}$ ), with intervals between administrations individualized to maintain sufficient IgG trough levels. CVID patients were vaccinated during the diagnostic process for suspected immunodeficiency with the Pneumo 23 vaccine (Polysaccharidum Streptococci pneumoniae type: $1,2,3,4,5,6 \mathrm{~B}, 7 \mathrm{~F}, 8,9 \mathrm{~N}, 9 \mathrm{~V}, 10 \mathrm{~A}, 11 \mathrm{~A}, 12 \mathrm{~F}, 14,15 \mathrm{~B}, 17 \mathrm{~F}$, $18 \mathrm{C}, 19 \mathrm{~A}, 19 \mathrm{~F}, 20,22 \mathrm{~F}, 23 \mathrm{~F}, 33 \mathrm{~F}$, and $25 \mu \mathrm{g}$ for each serotype provided by Sanofi Pasteur, Lyon, France) between 2 and 10 years before inclusion in this study. They showed lower than 4-fold increases in anti-PCP IgG 3-4 weeks after immunisation. CLL patients and healthy controls were never vaccinated with any antipneumococcal vaccine.

Before intravenous immunoglobulin administration, blood samples were collected from patients by venipuncture, allowed to clot naturally, and then the serum was separated and stored at $-80^{\circ} \mathrm{C}$. Each sample was thawed once and tested for total IgG, IgA, and IgM; anti-Gal IgG, IgA, and IgM; and anti-PCP IgG and IgA in all groups. Anti-PCP IgG2 was only assessed in controls and the B-CLL group. The ratio of serum anti-Gal $\operatorname{IgG}, \operatorname{IgA}$, and $\operatorname{IgM}(\mathrm{U} / \mathrm{mL})$ to the corresponding total serum $\operatorname{IgG}, \operatorname{IgA}$, or $\operatorname{IgM}(\mathrm{g} / \mathrm{L})$ in $\mathrm{U} / \mathrm{mg}$ and ratio of serum anti-PCP $\operatorname{IgG}$ and $\operatorname{IgA}(\mathrm{mg} / \mathrm{L})$ to the corresponding total serum $\operatorname{IgG}, \operatorname{IgA}$, or IgM were calculated in $\mathrm{mg} / \mathrm{L}$.

2.2. Serum IgG, IgA, and IgM Levels. Serum levels of IgG, IgA, and IgM were determined by immunonephelometry (Immage 800, Beckman-Coulter, Brea, CA, USA). Data were obtained in $\mathrm{g} / \mathrm{L}$.

2.3. Serum Anti-Gal IgG, IgA, and IgM Levels. The serum concentration of anti- $\alpha \mathrm{Gal}$ was determined by a sandwich enzyme-linked immunosorbent assay (ELISA), using an ELISA kit for human anti-alpha galactosyl IgG, IgA, and IgM (BioVendor, Laboratorní Medicína a.s., Brno, Czech Republic) according to the manufacturer's instructions. The 
TABLE 1: Characteristics of CLL cohort.

\begin{tabular}{|c|c|c|c|c|c|c|}
\hline Rai stage & 0 & I & II & III & IV & Total \\
\hline Patients' number & 14 & 8 & 14 & 15 & 8 & 59 \\
\hline IgVH mutated/unmutated/NA & $10 / 1 / 3$ & $3 / 2 / 3$ & $3 / 9 / 2$ & $1 / 9 / 5$ & $3 / 3 / 2$ & $20 / 24 / 15$ \\
\hline TP53 mutated/unmutated/NA & $0 / 6 / 8$ & $0 / 5 / 3$ & $0 / 12 / 2$ & $1 / 8 / 6$ & $0 / 5 / 3$ & $1 / 36 / 22$ \\
\hline Normal karyotype & 3 & 0 & 1 & 5 & 1 & 10 \\
\hline $13 q$ deletion & 7 & 1 & 4 & 3 & 0 & 15 \\
\hline 12 trisomy & 0 & 1 & 2 & 4 & 3 & 10 \\
\hline 11q deletion & 0 & 2 & 2 & 1 & 2 & 7 \\
\hline $17 \mathrm{p}$ deletion & 0 & 0 & 1 & 0 & 0 & 1 \\
\hline Other karyotype changes & 1 & 3 & 4 & 2 & 1 & 11 \\
\hline No cytogenetics available & 3 & 1 & 0 & 0 & 1 & 5 \\
\hline Sinusitis & 0 & 0 & 2 & 0 & 0 & 2 \\
\hline Bronchitis & 2 & 0 & 0 & 0 & 0 & 2 \\
\hline Pneumonia & 0 & 0 & 1 & 0 & 1 & 2 \\
\hline Other bacterial infection requiring ATB therapy & 2 & 1 & 1 & 2 & 2 & 8 \\
\hline Hospitalisation due to infection & 0 & 0 & 1 & 1 & 1 & 3 \\
\hline
\end{tabular}

IgVH: immunoglobulin heavy chain variable region gene; TP53: tumour protein 53; NA: data not available. Only prognostically worst karyotype change is recorded; all infections were recorded in the period of one year before blood sample collection.

range of detection was 3.13-100 U/mL for every kit. Serum samples were diluted $100 \mathrm{x}$, and absorbance was read at $450 \mathrm{~nm}$ using a Multiskan RC ELISA reader (Thermo Fisher Scientific, Waltham, MA, USA).

2.4. Serum Anti-PCP IgG, IgG2, and IgA Levels. The serum levels of anti-PCP were determined by ELISA using specific VaccZyme $^{\mathrm{TM}}$ ELISA kits for human anti-PCP IgG, IgG2, and IgA (Binding Site, Birmingham, UK), according to the manufacturer's instructions. The range of detection was 3.3-270 $\mathrm{mg} / \mathrm{L}$ for the IgG kit, $1.1-90 \mathrm{mg} / \mathrm{L}$ for the IgG2 kit, and $0-270 \mathrm{U} / \mathrm{mL}$ for the IgA kit. Serum samples were diluted $100 \mathrm{x}$, and the absorbance was read at $450 \mathrm{~nm}$ using a Multiskan RC ELISA reader (Thermo Fisher Scientific).

2.5. Data Analysis. Nonparametric tests were used to analyse the data because of insufficient numbers of observations and skewed readings. Spearman's rank correlation coefficient was used to determine the dependency of each parameter on age and, for CLL patients, disease stage. The KruskalWallis test was used to compare all groups, followed by the Wilcoxon two-sample rank-sum test to compare each group by two when the Kruskal-Wallis test rejected the null hypothesis. All $p$ values were adjusted using Bonferroni correction, and $p<0.05$ was considered significant. Statistical analysis and other computations were performed using $\mathrm{R}$ language (Core Team, Vienna, Austria, 2016)

\section{Results}

Descriptive statistical data for all parameters are shown in Tables 2 and 3. There was no statistically significant correlation with age for any parameters in our cohort (data not shown). Comparisons between groups are shown in Table 4 and Figures 1, 2, and 3 using box plots with the median, first and the third quartiles, and whiskers with 1.5 times the interquartile range marked with the statistically significant pairs.

In the CLL group, we observed a decrease below the normal range for age in at least one of the immunoglobulin classes in 38/59 (64.4\%) CLL patients: $\operatorname{lgG}<7.3 \mathrm{~g} / \mathrm{L}$ in $18 / 59$ (30.5\%) patients; IgA $<0.8 \mathrm{~g} / \mathrm{L}$ in $27 / 59$ (45.7\%) patients; and $\operatorname{IgM}<0.4 \mathrm{~g} / \mathrm{L} \mathrm{28/59}$ (47.5\%) patients. An IgG level less than $4 \mathrm{~g} / \mathrm{L}$ was only observed in $4 / 59(6.7 \%)$ patients. Although, anti-PCP IgG and anti-PCP IgG2 (CLL) did not differ significantly from controls, 15/59 (25.4\%) CLL patients had anti-PCP IgG lower than $20 \mathrm{U} / \mathrm{mL}$. The levels of $\operatorname{IgA}(p=0.0118 ; r=-0.4306)$, anti-Gal IgA $(p=0.0286 ; r=-0.3982)$, anti-PCP $\operatorname{IgA}(p=0.0085 ; r=$ $-0.4374)$, and anti-PCP $\operatorname{IgG} 2(p=0.0363 ; r=-0.3898)$ were negatively correlated with disease stage.

The ratio to the serum immunoglobulin level was measured. In CVID patients, the ratio of anti-PCP IgG $(p<0.00001)$ to serum IgG level was significantly higher than that in controls. In the CLL group, the ratios of anti-Gal IgA $(p=0.0004)$ and anti-Gal IgM $(p=0.0012)$ to total IgA/IgM were already lower than those in controls. No significant difference in the anti-Gal IgG to total IgG ratio in all groups and anti-PCP IgG2 and IgA to total IgG/IgA ratios between CLL patients and controls (data not shown). In CVID group, this comparison was not made for $\operatorname{IgA}$ and $\operatorname{IgM}$ because of the significant number of small values in most parameters in CVID patients.

\section{Discussion}

Hypogammaglobulinaemia is the most common immunodeficiency in CLL and occurs in more than $85 \%$ patients at some point during the disease [5] and in 25\% of newly diagnosed patients [21]. Its prevalence and extent correlate with disease duration, advancing stage, and infection frequency [21-26]. Hypogammaglobulinaemia occurs in $10 \%$ 
TABLE 2: Descriptive statistics (all groups).

\begin{tabular}{lccccccccc}
\hline & \multicolumn{3}{c}{ CLL $(n=59)$} & \multicolumn{3}{c}{ CVID $(n=30)$} & \multicolumn{3}{c}{ Controls $(n=67)$} \\
& Median & Q1 & Q3 & Median & Q1 & Q3 & Median & Q1 & Q3 \\
\hline IgG (g/L) & 8.26 & 5.84 & 10.75 & 6.85 & 5.80 & 7.88 & 12.80 & 10.75 & 14.95 \\
IgA (g/L) & 0.82 & 0.48 & 1.69 & 0.07 & 0.07 & 0.14 & 2.58 & 1.96 & 3.12 \\
IgM (g/L) & 0.46 & 0.26 & 0.81 & 0.11 & 0.07 & 0.20 & 1.6 & 0.84 & 1.42 \\
Anti-Gal IgG (U/mL) & 45.88 & 13.69 & 80.21 & 31.60 & 27.50 & 39.50 & 64.01 & 30.80 & 144.90 \\
Anti-Gal IgA (U/mL) & 5.35 & 2.93 & 11.16 & 0.01 & 0.01 & 1.50 & 25.00 & 16.12 & 36.80 \\
Anti-Gal IgM (U/mL) & 5.70 & 1.97 & 11.31 & 1.60 & 0.50 & 6.68 & 27.80 & 14.90 & 48.68 \\
Anti-PCP IgG (mg/L) & 41.10 & 19.9 & 68.17 & 65.05 & 52.70 & 79.47 & 58.30 & 30.76 & 108.70 \\
Anti-PCP IgG2 (mg/L) & 13.73 & 7.5 & 24.49 & - & - & - & 15.24 & 7.26 & 33.10 \\
Anti-PCP IgA (mg/L) & 7.58 & 4.16 & 17.52 & 0.63 & 0.20 & 2.18 & 27.00 & 12.50 & 55.65 \\
\hline
\end{tabular}

Because the data were not normally distributed, they are shown as the median, Q1 (first quartile), and Q3 (third quartile).

TABle 3: Descriptive statistics CLL (Rai staging).

\begin{tabular}{|c|c|c|c|c|c|c|}
\hline Stadium (Rai) & 0 & I & II & III & IV & Total \\
\hline \multirow{2}{*}{$\operatorname{IgG}(\mathrm{g} / \mathrm{L})$} & 10.14 & 6.80 & 8.22 & 7.94 & 5.80 & 8.26 \\
\hline & $(8.68 ; 13.18)$ & $(5.36 ; 8.82)$ & $(7.08 ; 10.07)$ & $(6.29 ; 11.15)$ & $(4.15 ; 7.64)$ & $(5.84 ; 10.75)$ \\
\hline \multirow{2}{*}{$\operatorname{IgA}(g / L)$} & 1.75 & 0.43 & 1.21 & 0.73 & 0.45 & 0.82 \\
\hline & $(1.17 ; 2.09)$ & $(0.38 ; 0.53)$ & $(0.76 ; 1.72)$ & $(0.49 ; 0.91)$ & $(0.35 ; 0.83)$ & $(0.48 ; 1.69)$ \\
\hline \multirow{2}{*}{$\operatorname{IgM}(g / L)$} & 0.79 & 0.29 & 0.43 & 0.30 & 0.24 & 0.46 \\
\hline & $(0.57 ; 0.83)$ & $(0.21 ; 0.45)$ & $(0.33 ; 0.65)$ & $(0.20 ; 0.62)$ & $(0.12 ; 0.47)$ & $(0.26 ; 0.81)$ \\
\hline \multirow{2}{*}{ Anti-Gal IgG (U/L) } & 65.32 & 35.80 & 29.70 & 49.78 & 10.42 & 45.88 \\
\hline & $(18.73 ; 132.10)$ & $(2.73 ; 50.51)$ & $(0.78 ; 57.09)$ & $(4.38 ; 85.90)$ & $(1.78 ; 86.15)$ & $(13.69 ; 80.21)$ \\
\hline \multirow{2}{*}{ Anti-Gal IgA (U/mL) } & 11.80 & 2.37 & 5.9 & 4.70 & 2.84 & 5.35 \\
\hline & $(8.23 ; 16.39)$ & $(1.56 ; 6.31)$ & $(3.05 ; 8.33)$ & $(3.38 ; 9.19)$ & $(1.07 ; 5.19)$ & $(2.93 ; 11.16)$ \\
\hline \multirow{2}{*}{ Anti-Gal IgM (U/mL) } & 10.22 & 9.41 & 3.74 & 6.87 & 1.89 & 5.7 \\
\hline & $(6.19 ; 20.05)$ & $(2.42 ; 13.97)$ & $(1.75 ; 7.57)$ & $(1.59 ; 11.96)$ & $(1.25 ; 3.35)$ & $(1.97 ; 11.31)$ \\
\hline \multirow{2}{*}{ Anti-PCP IgG (mg/L) } & 62.66 & 45.72 & 30.4 & 42.58 & 20.86 & 41.10 \\
\hline & $(38.18 ; 89.64)$ & $(36.57 ; 57.67)$ & $(15.69 ; 56.06)$ & $(15.42 ; 73.97)$ & $(17.32 ; 37.50)$ & $(19.09 ; 68.17)$ \\
\hline \multirow{2}{*}{ Anti-PCP IgG2 (mg/L) } & 24.28 & 19.10 & 10.86 & 10.19 & 7.14 & 13.73 \\
\hline & $(13.96 ; 29.86)$ & $(14.80 ; 23.43)$ & $(5.32 ; 18.38)$ & $(4.48 ; 21.35)$ & $(6.60 ; 11.43)$ & $(7.05 ; 24.49)$ \\
\hline \multirow{2}{*}{ Anti-PCP IgA (mg/L) } & 15.64 & 6.41 & 10.57 & 6.97 & 3.74 & 7.58 \\
\hline & $(9.62 ; 22.58)$ & $(4.20 ; 10.30)$ & $(5.65 ; 24.78)$ & $(3.79 ; 9.37)$ & $(3.23 ; 4.67)$ & $(4.16 ; 17.52)$ \\
\hline
\end{tabular}

Because the data were not normally distributed, they are shown as the median, Q1 (first quartile), and Q3 (third quartile).

TABLE 4: Comparison between groups.

\begin{tabular}{lccc}
\hline & CLL $\times$ controls & CLL $\times$ CVID & CVID $\times$ controls \\
\hline IgG & $<0.0001$ & 1 & $<0.0001$ \\
IgA & $<0.0001$ & $<0.0001$ & $<0.0001$ \\
IgM & $<0.0001$ & 0.0001 & $<0.0001$ \\
Anti-Gal IgG & 0.3303 & 1 & 0.0180 \\
Anti-Gal IgA & $<0.0001$ & 0.0002 & $<0.0001$ \\
Anti-Gal IgM & $<0.0001$ & 0.3504 & $<0.0001$ \\
Anti-PCP IgG & $\mathrm{ND}$ & $\mathrm{ND}$ & $\mathrm{ND}$ \\
Anti-PCP IgG2 & $\mathrm{ND}$ & $\mathrm{ND}$ & $\mathrm{ND}$ \\
Anti-PCP IgA & $<0.0001$ & $<0.0001$ & $<0.0001$ \\
\hline
\end{tabular}

ND: Wilcoxon two-sample rank-sum test was not performed because the $p$ value was more than 0.05 in Kruskal-Wallis test. of patients with Binet stage A [27] and up to $100 \%$ of patients with Binet stage C [28]. Its aetiology is multifactorial because of a combination of disease-related immune defects and iatrogenic immunosuppression that affects both humoral and cellular immunity $[5,25]$.

The prognostic significance of hypogammaglobulinaemia in terms of morbidity, mortality, and overall survival of CLL patients remains controversial. In a study by Rozman et al., the only immunoglobulin class associated with shorter survival in multivariate analysis was IgA [29]. Similarly, in a study by Shvidel et al., IgA was associated with shortened survival, but only in univariate analysis. In multivariate analysis, there was no association between survival and any immunoglobulin class [27]. Andersen et al. described the negative prognostic impact of hypogammaglobulinaemia in 


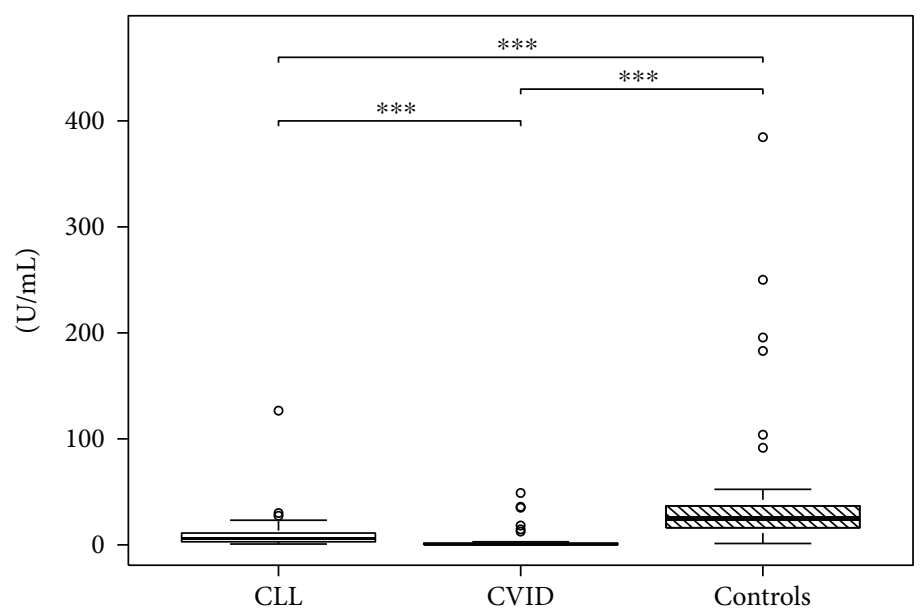

FIgURE 1: Comparison of anti-Gal IgA (U/mL) in peripheral blood. Results are presented as box plots with the median, first and third quartiles, and whiskers with 1.5 times the interquartile range marked with statistically significant pairs of groups. All groups showed significant differences $\left(p^{* * *}<0.001\right)$ for anti-Gal IgA.

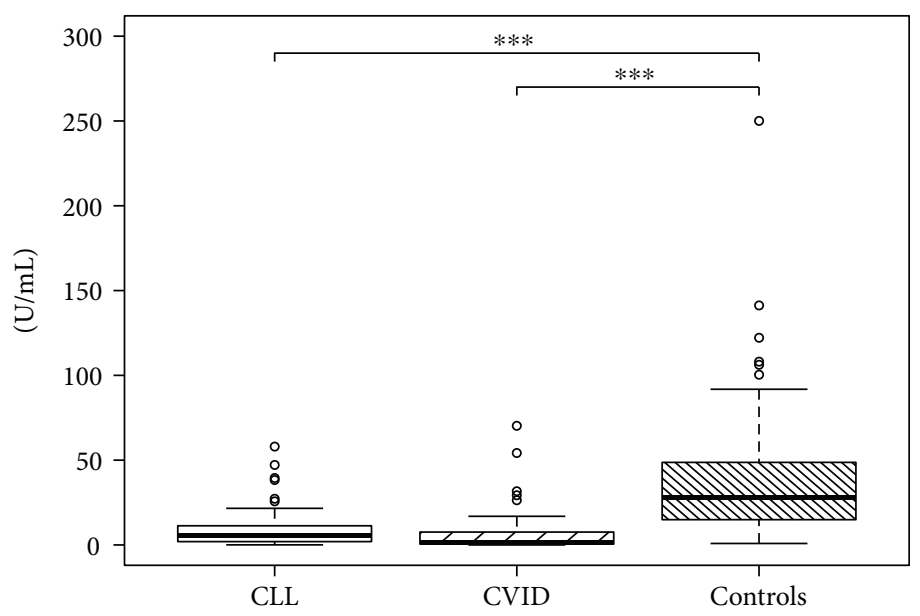

Figure 2: Comparison of anti-Gal IgM (U/mL) in peripheral blood. Results are presented as box plots with the median, first and third quartiles, and whiskers with 1.5 times the interquartile range marked with statistically significant pairs of groups. CLL and CVID groups showed significantly lower anti-Gal IgM level than healthy controls did $\left(p^{* * *}<0.001\right)$.

all classes on overall survival. However, the largest study on this topic conducted at the Mayo Clinic revealed no such association (although this study tested only IgG) [21]. Morrison et al. focused not on overall survival but on the frequency of infectious complications. In this study, only the levels of IgA were found to be significant [30].

Whereas immunoglobulin substitution therapy is indicated strictly in all CVIDs, in secondary immunodeficiencies, there is a lack of specific markers for identifying patients at higher risk of infections and for determining preemptive IgG replacement therapy. An Italian multicentre prospective cohort study identified specific CVID clinical phenotype characterised by a high pneumonia risk: low IgG and IgA levels at the time of diagnosis, an IgA level $<7 \mathrm{mg} / \mathrm{dL}$, and bronchiectasis [31]. The same increased risk was identified in poor responders to the 23 -valent pneumococcal polysaccharide vaccine in the IgA class [32].
Notably, some CLL patients with sufficient immunoglobulin concentrations suffer from recurrent infections, whereas some with hypogammaglobulinaemia do not. Therefore, not only the quantity of immunoglobulins but also the relative levels of IgG subclasses $[33,34]$ and the ability potential of $B$ cells to form specific immune response are important [35]. Vinsentin et al. examined the best protective cutoff for each immunoglobulin isotype across disease stages, regardless of the therapy provided, with the following results: $7.44 \mathrm{~g} / \mathrm{L}$ for $\operatorname{IgG}, 0.79 \mathrm{~g} / \mathrm{L}$ for $\mathrm{IgA}$, and $0.21 \mathrm{~g} / \mathrm{L}$ for $\operatorname{IgM}[22]$.

As expected, we observed lower levels of all immunoglobulin classes in CLL patients than in healthy controls. However, most patients had IgG levels within the normal range (69.5\%), and only $6.7 \%$ had IgG levels below $4 \mathrm{~g} / \mathrm{L}$. This was expected because a significant proportion of patients had early or indolent disease, and none had been treated for their disease at the time of blood sample collection. 


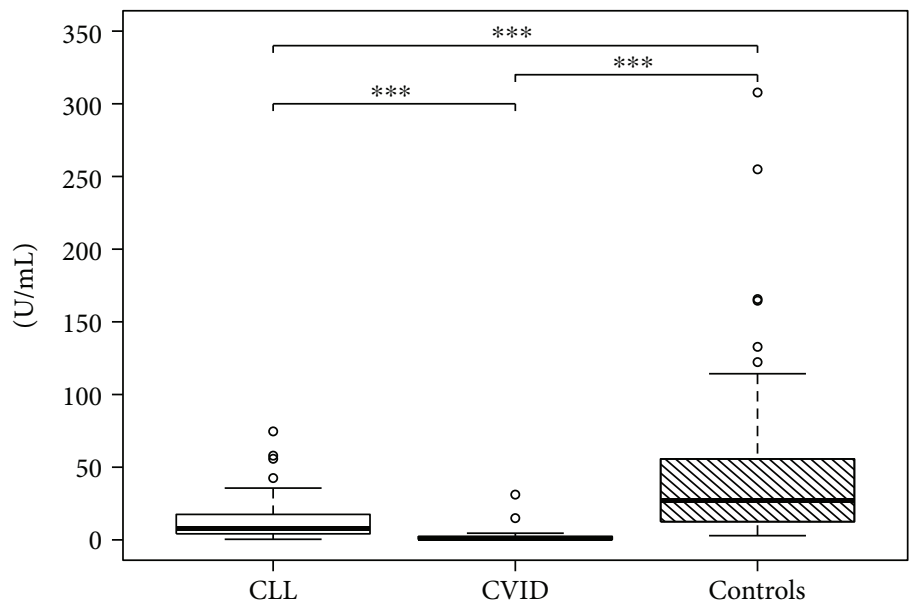

FIgURE 3: Comparison of anti-PCP IgA (mg/L) in peripheral blood. Results are presented as box plots with the median, first and third quartiles, and whiskers with 1.5 times the interquartile range marked with statistically significant pairs of groups. All groups showed significant differences $\left(p^{* * *}<0.001\right)$ in anti-PCP IgA.

Serious bacterial infections (requiring hospitalization and/or administration of intravenous antibiotics) in our CLL cohort were observed only in 3 of 59 (5\%) patients. Thus, we considered analysis of the causal link with immunoglobulin levels statistically meaningless. Although the decreased levels of IgG and anti-PCP IgG did not differ from those in healthy controls, only 12 of 59 (20\%) CLL patients had been treated with one $(9 / 59,15 \%)$ or more $(3 / 59,5 \%)$ antibiotic courses due to bacterial infection in the last year. Thus, despite their lower IgG levels and higher anti-PCP levels, these patients had fewer infections. In contrast, low levels of pneumococcal antibodies are associated with severe or multiple infections [23].

Antipneumococcal vaccination is recommended in CLL patients because $S$. pneumoniae is considered a dominant pathogen related to humoral immunodeficiency [11]. The response against polysaccharide antigens may not be sufficient in CLL patients, while conjugate vaccines appear to be more effective [36]. Pasiarski et al. found a statistically significant increase in the titres of specific antipneumococcal IgG following administration of 13-valent pneumococcal conjugate vaccine in CLL patients, although the titres were still much lower in the CLL group than in the control group [28]. Previous studies showed that vaccination can suppress disease progression $[37,38]$. B-CLL patients in our study were not vaccinated. Their anti-PCP IgG and anti-PCP IgG2 levels were similar to that of healthy controls, but anti-PCP IgG2 was negatively correlated with disease stage. Although anti-PCP IgG reflects immunoglobulin substitution therapy in CVIDs, anti-PCP IgA levels in this group corresponded with primary immunodeficiency.

There may be a very important vaccination response not only in the IgG class but also in IgA. Our original data revealed lower levels of anti-PCP IgA in nonvaccinated CLL patients than in healthy controls. Total IgA and anti-PCP IgA were negatively correlated with disease stage. The postvaccination response in the IgA class remains unknown. It was not surprising that anti-PCP IgA level in CVID patients was lower than that in healthy controls and the CLL cohort because of more profound defects in humoral immunity.

Re-evaluation of actual antibody production is limited after initiation of substitution immunoglobulin therapy. Specific anti-PCP IgA responses and measured titres of isohaemagglutinins (naturally occurring antibodies mainly of IgM isotypes to polysaccharide blood group antigens) may be an alternative clinically relevant method for assessing $\mathrm{T}$ cell-independent antibody production in patients who have already been started on IgG therapy [12].

Natural anti-Gal antibodies represent another helpful marker which can be exploited in assessing antibody production ability. The possibility of determining levels of not only anti-Gal IgG, but also IgA and IgM, allows for evaluation in immunoglobulin-substituted patients. In contrast to specific antipneumococcal antibodies produced in relatively low amount, regarding the specificity, anti-Gal is the most abundant natural antibody in humans. As many as $1 \%$ of human B cells can produce anti-Gal, and those along the gastrointestinal tract produce this antibody in response to continuous antigenic stimulation by gastrointestinal bacteria [15]. Mucosal stimulation and production of antibodies in classes IgM as well as IgA are important in defence against infections. These antibodies could serve for assessment of specific humoral immune response in immunodeficient patients.

Little is known regarding anti-Gal production in immunodeficient patients. Subjects with defects in T celldependent antibody synthesis may have normal levels of xenoreactive natural antibodies, most of which are specific for Gal alpha 1-3 Gal. Parker et al. described the case of one agammaglobulinaemic patient with undetectable antiGal IgM concentration, three patients with severe combined immunodeficiency, and four Wiskott-Aldrich patients with the same findings [39].

We present here the first report of a cohort of 30 CVID patients treated with immunoglobulin substitution therapy and 59 CLL nonsubstituted patients with confirmed decrease in anti-Gal IgA and IgM. The decrease likely depended on the 
severity of the immunity defect. The level of anti-Gal IgA in the CLL cohort was negatively correlated with disease stage. IgG and anti-Gal IgG levels may be influenced by immunoglobulin substitution therapy. We found no difference between the CVID and CLL groups in the levels of anti-Gal IgM. Our CLL patients were laboratory assessed early after the clinical diagnosis with only low numbers of infections in all clinical stages (Table 1). Therefore, the longer time of follow-up and recruitment of new CLL patients will allow the statistical correlation analysis regarding sinusitis, bronchitis, and pneumonia, thus requiring $\mathrm{ATB}$ and the ratio of anti-Gal IgA and IgM to total IgA and IgM, respectively. This is the next point for further investigations.

Moreover, our data suggest that anti-Gal IgA and antiGal IgM are highly sensitive markers that can be used to investigate the ability of specific antibody production in patients with primary and secondary immunodeficiency. The results for both isotypes (IgA and $\operatorname{IgM}$ ) agreed with those for anti-PCP antibodies in immunodeficient patients ([32, 40], Figure 3). Notably, immunization of patients is not required for anti-Gal antibody production, and thus the examination is vaccine-independent and noninvasive.

\section{Conclusions}

Evaluation of antibody production plays a key role in the diagnosis and management of humoral immunodeficiencies. We suggest the use of anti-Gal antibodies in routine testing as an alternative to isohaemagglutinins for the diagnosis of hypogammaglobulinaemia. Significant reductions mainly in anti-Gal IgA, anti-Gal IgM, and anti-PCP IgA may have prognostic importance in immunodeficiency related to CLL. These tests are suitable even in cases of regular immunoglobulin substitution therapy.

\section{Conflicts of Interest}

The authors declare that there are no conflicts of interest regarding the publication of this paper.

\section{Acknowledgments}

This work was supported by Charles University in Prague, Faculty of Medicine in Hradec Kralove, Czech Republic, Project "PRVOUK" P37/10 and PROGRES 2017, CETOCOEN PLUS, CZ.02.1.01/0.0/0.0/15_003/0000469 of MEYS (The Ministry of Education, Youth and Sports).

\section{References}

[1] L. Scarfo, A. J. Ferreri, and P. Ghia, "Chronic lymphocytic leukaemia," Critical Reviews in Oncology/Hematology, vol. 104, pp. 169-182, 2016.

[2] F. Van Bockstaele, B. Verhasselt, and J. Philippé, "Prognostic markers in chronic lymphocytic leukemia: a comprehensive review," Blood Reviews, vol. 23, no. 1, pp. 25-47, 2009.

[3] C. A. Dasanu and D. T. Alexandrescu, "Risk for second nonlymphoid neoplasms in chronic lymphocytic leukemia," Medscape General Medicine, vol. 9, no. 4, p. 35, 2007.
[4] S. Molica, "Infections in chronic lymphocytic leukemia: risk factors, and impact on survival, and treatment," Leukemia \& Lymphoma, vol. 13, no. 3-4, pp. 203-214, 1994.

[5] D. Oscier, C. Dearden, E. Eren et al., "Guidelines on the diagnosis, investigation and management of chronic lymphocytic leukaemia," British Journal of Haematology, vol. 159, no. 5, pp. 541-564, 2012.

[6] A. D. Hamblin and T. J. Hamblin, "The immunodeficiency of chronic lymphocytic leukaemia," British Medical Bulletin, vol. 87, pp. 49-62, 2008.

[7] F. Ravandi and S. O'Brien, "Immune defects in patients with chronic lymphocytic leukemia," Cancer Immunology, Immunotherapy, vol. 55, no. 2, pp. 197-209, 2006.

[8] C. Dearden, "Disease-specific complications of chronic lymphocytic leukemia," Hematology. American Society of Hematology. Education Program, vol. 2008, pp. 450-456, 2008.

[9] V. Friman, O. Winqvist, C. Blimark, P. Langerbeins, H. Chapel, and F. Dhalla, "Secondary immunodeficiency in lymphoproliferative malignancies," Hematological Oncology, vol. 34, no. 3, pp. 121-132, 2016.

[10] S. Sanchez-Ramon, F. Dhalla, and H. Chapel, "Challenges in the role of gammaglobulin replacement therapy and vaccination strategies for hematological malignancy," Frontiers in Immunology, vol. 7, p. 317, 2016.

[11] S. Jolles, H. Chapel, and J. Litzman, "When to initiate immunoglobulin replacement therapy (IGRT) in antibody deficiency: a practical approach," Clinical and Experimental Immunology, vol. 188, no. 3, pp. 333-341, 2017.

[12] F. A. Bonilla, I. Barlan, H. Chapel et al., "International Consensus Document (ICON): common variable immunodeficiency disorders," The Journal of Allergy and Clinical Immunology. In Practice, vol. 4, no. 1, pp. 38-59, 2016.

[13] E. S. Resnick, E. L. Moshier, J. H. Godbold, and C. Cunningham-Rundles, "Morbidity and mortality in common variable immune deficiency over 4 decades," Blood, vol. 119, no. 7, pp. 1650-1657, 2012.

[14] B. Gathmann, N. Mahlaoui, CEREDIH et al., "Clinical picture and treatment of 2212 patients with common variable immunodeficiency," The Journal of Allergy and Clinical Immunology, vol. 134, no. 1, pp. 116-126, 2014.

[15] U. Galili, "Anti-Gal: an abundant human natural antibody of multiple pathogeneses and clinical benefits," Immunology, vol. 140, no. 1, pp. 1-11, 2013.

[16] H. Saleh, S. Embry, A. Nauli, S. Atyia, and G. Krishnaswamy, "Anaphylactic reactions to oligosaccharides in red meat: a syndrome in evolution," Clinical and Molecular Allergy, vol. 10, no. 1, p. 5, 2012.

[17] C. H. Chung, B. Mirakhur, E. Chan et al., "Cetuximab-induced anaphylaxis and IgE specific for galactose- $\alpha$-1,3-galactose," The New England Journal of Medicine, vol. 358, no. 11, pp. 1109-1117, 2008.

[18] M. Hamanova, M. Chmelikova, I. Nentwich, V. Thon, and J. Lokaj, "Anti-Gal IgM, IgA and IgG natural antibodies in childhood," Immunology Letters, vol. 164, no. 1, pp. 40-43, 2015.

[19] J. M. Bernth-Jensen, B. K. Moller, J. C. Jensenius, and S. Thiel, "Biological variation of anti-alpha Gal-antibodies studied by a novel time-resolved immunofluorometric assay," Journal of Immunological Methods, vol. 373, no. 1-2, pp. 26-35, 2011.

[20] M. E. Conley, L. D. Notarangelo, and A. Etzioni, "Diagnostic criteria for primary immunodeficiencies. representing PAGID 
(Pan-American Group for Immunodeficiency) and ESID (European Society for Immunodeficiencies)," Clinical Immunology, vol. 93, no. 3, pp. 190-197, 1999.

[21] S. A. Parikh, J. F. Leis, K. G. Chaffee et al., "Hypogammaglobulinemia in newly diagnosed chronic lymphocytic leukemia: natural history, clinical correlates, and outcomes," Cancer, vol. 121, no. 17, pp. 2883-2891, 2015.

[22] A. Visentin, N. Compagno, F. Cinetto et al., "Clinical profile associated with infections in patients with chronic lymphocytic leukemia. Protective role of immunoglobulin replacement therapy," Haematologica, vol. 100, no. 12, pp. e515-e518, 2015.

[23] H. Griffiths, J. Lea, C. Bunch, M. Lee, and H. Chapel, "Predictors of infection in chronic lymphocytic leukaemia (CLL)," Clinical and Experimental Immunology, vol. 89, no. 3, pp. 374-377, 1992.

[24] I. Ben-Bassat, A. Many, M. Modan, C. Peretz, and B. Ramot, "Serum immunoglobulins in chronic lymphocytic leukemia," The American Journal of the Medical Sciences, vol. 278, no. 1, pp. 4-9, 1979.

[25] V. A. Morrison, "Infectious complications of chronic lymphocytic leukaemia: pathogenesis, spectrum of infection, preventive approaches," Best Practice \& Research. Clinical Haematology, vol. 23, pp. 145-153, 2010.

[26] M. A. Andersen, F. J. Vojdeman, M. K. Andersen et al., "Hypogammaglobulinemia in newly diagnosed chronic lymphocytic leukemia is a predictor of early death," Leukemia \& Lymphoma, vol. 57, no. 7, pp. 1592-1599, 2016.

[27] L. Shvidel, T. Tadmor, A. Braester et al., "Serum immunoglobulin levels at diagnosis have no prognostic significance in stage A chronic lymphocytic leukemia: a study of 1113 cases from the Israeli CLL study group," European Journal of Haematology, vol. 93, no. 1, pp. 29-33, 2014.

[28] M. Pasiarski, J. Rolinski, E. Grywalska et al., "Antibody and plasmablast response to 13 -valent pneumococcal conjugate vaccine in chronic lymphocytic leukemia patients - preliminary report," PLoS One, vol. 9, no. 12, article e114966, 2014.

[29] C. Rozman, E. Montserrat, and N. Viñolas, "Serum immunoglobulins in B-chronic lymphocytic leukemia. Natural history and prognostic significance," Cancer, vol. 61, no. 2, pp. 279283, 1988.

[30] P. D. Wadhwa and V. A. Morrison, "Infectious complications of chronic lymphocytic leukemia," Seminars in Oncology, vol. 33, pp. 240-249, 2006.

[31] I. Quinti, A. Soresina, A. Guerra et al., "Effectiveness of immunoglobulin replacement therapy on clinical outcome in patients with primary antibody deficiencies: results from a multicenter prospective cohort study," Journal of Clinical Immunology, vol. 31, no. 3, pp. 315-322, 2011.

[32] F. M. Cavaliere, C. Milito, H. Martini et al., "Quantification of IgM and IgA anti-pneumococcal capsular polysaccharides by a new ELISA assay: a valuable diagnostic and prognostic tool for common variable immunodeficiency," Journal of Clinical Immunology, vol. 33, no. 4, pp. 838-846, 2013.

[33] J. A. Freeman, K. R. Crassini, O. G. Best et al., "Immunoglobulin $G$ subclass deficiency and infection risk in 150 patients with chronic lymphocytic leukemia," Leukemia \& Lymphoma, vol. 54, no. 1, pp. 99-104, 2013.

[34] T. Svensson, M. Hoglund, and H. Cherif, "Clinical significance of serum immunoglobulin $G$ subclass deficiency in patients with chronic lymphocytic leukemia," Scandinavian Journal of Infectious Diseases, vol. 45, pp. 537-542, 2013.
[35] A. Nosari, "Infectious complications in chronic lymphocytic leukemia," Mediterranean Journal of Hematology and Infectious Diseases, vol. 4, no. 1, article e2012070, 2012.

[36] M. Sinisalo, J. Aittoniemi, P. Oivanen, H. Käyhty, R. M. Olander, and J. Vilpo, "Response to vaccination against different types of antigens in patients with chronic lymphocytic leukaemia," British Journal of Haematology, vol. 114, no. 1, pp. 107-110, 2001.

[37] A. Hartkamp, A. H. Mulder, G. T. Rijkers, H. van Velzen-Blad, and D. H. Biesma, "Antibody responses to pneumococcal and haemophilus vaccinations in patients with B-cell chronic lymphocytic leukaemia," Vaccine, vol. 19, no. 13-14, pp. 1671-1677, 2001.

[38] M. Sinisalo, J. Vilpo, M. Itälä, M. Väkeväinen, J. Taurio, and J. Aittoniemi, “Antibody response to 7-valent conjugated pneumococcal vaccine in patients with chronic lymphocytic leukaemia," Vaccine, vol. 26, no. 1, pp. 82-87, 2007.

[39] W. Parker, K. Lundberg-Swanson, Z. E. Holzknecht et al., "Isohemagglutinins and xenoreactive antibodies: members of a distinct family of natural antibodies," Human Immunology, vol. 45, no. 2, pp. 94-104, 1996.

[40] Z. Chovancova, M. Vlkova, J. Litzman, J. Litzman, J. Lokaj, and V. Thon, "Antibody forming cells and plasmablasts in peripheral blood in CVID patients after vaccination," Vaccine, vol. 29, no. 24, pp. 4142-4150, 2011. 


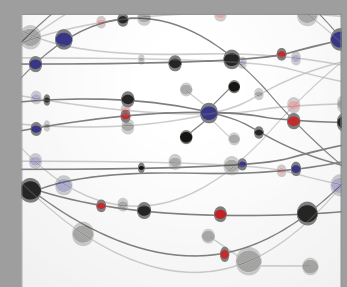

The Scientific World Journal
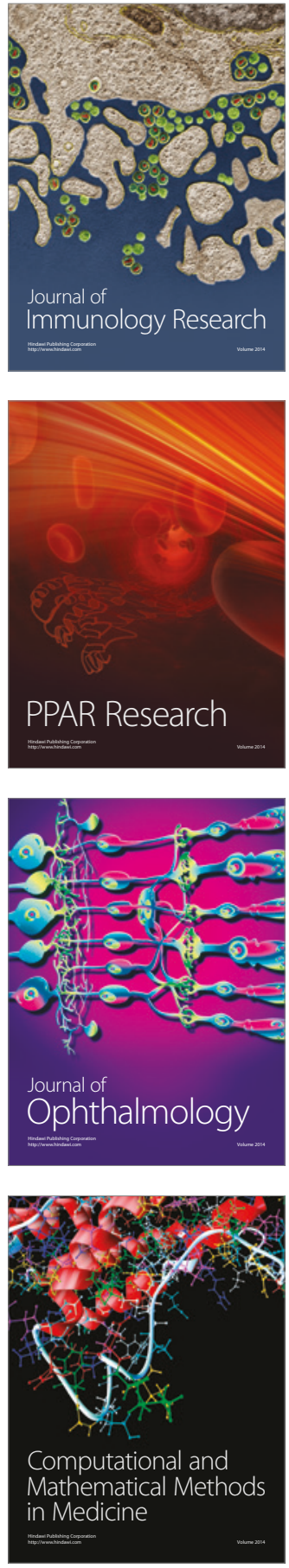

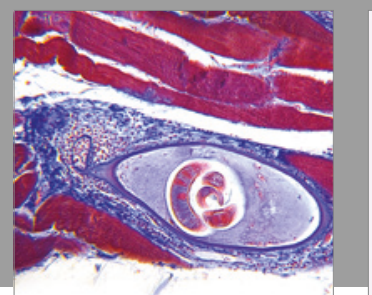

Gastroenterology Research and Practice
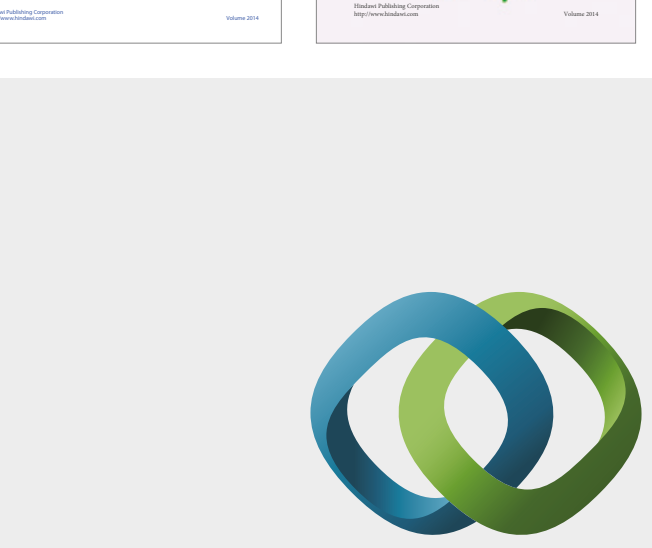

\section{Hindawi}

Submit your manuscripts at

https://www.hindawi.com
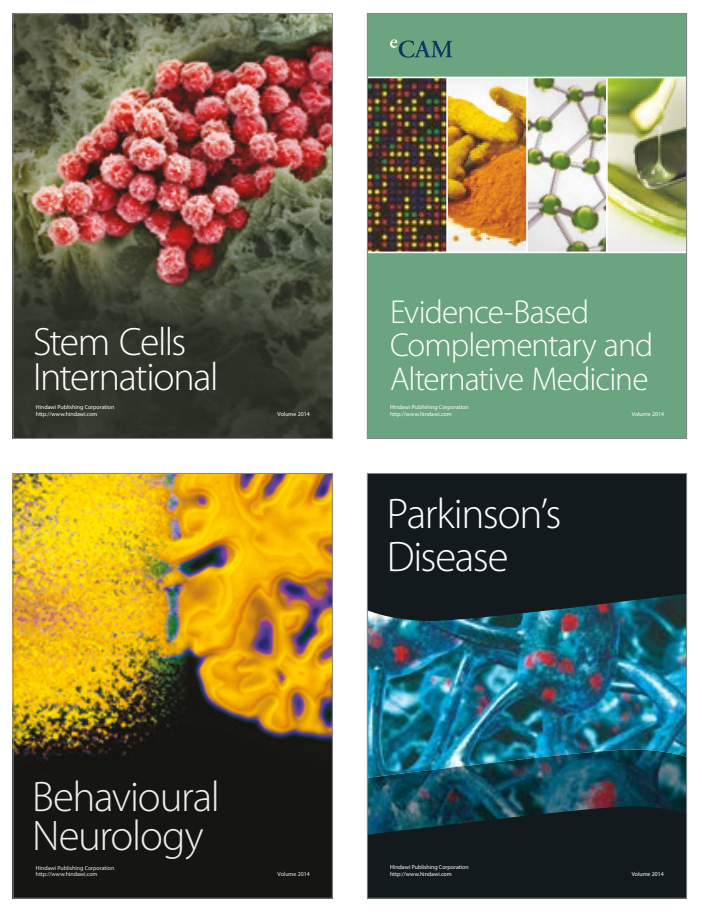
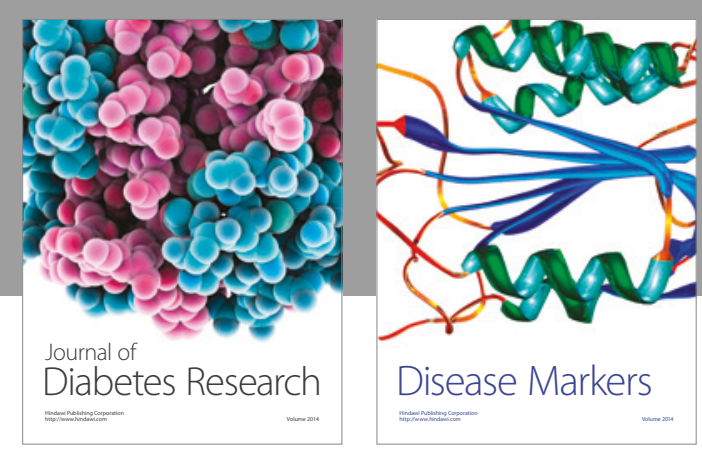

Disease Markers
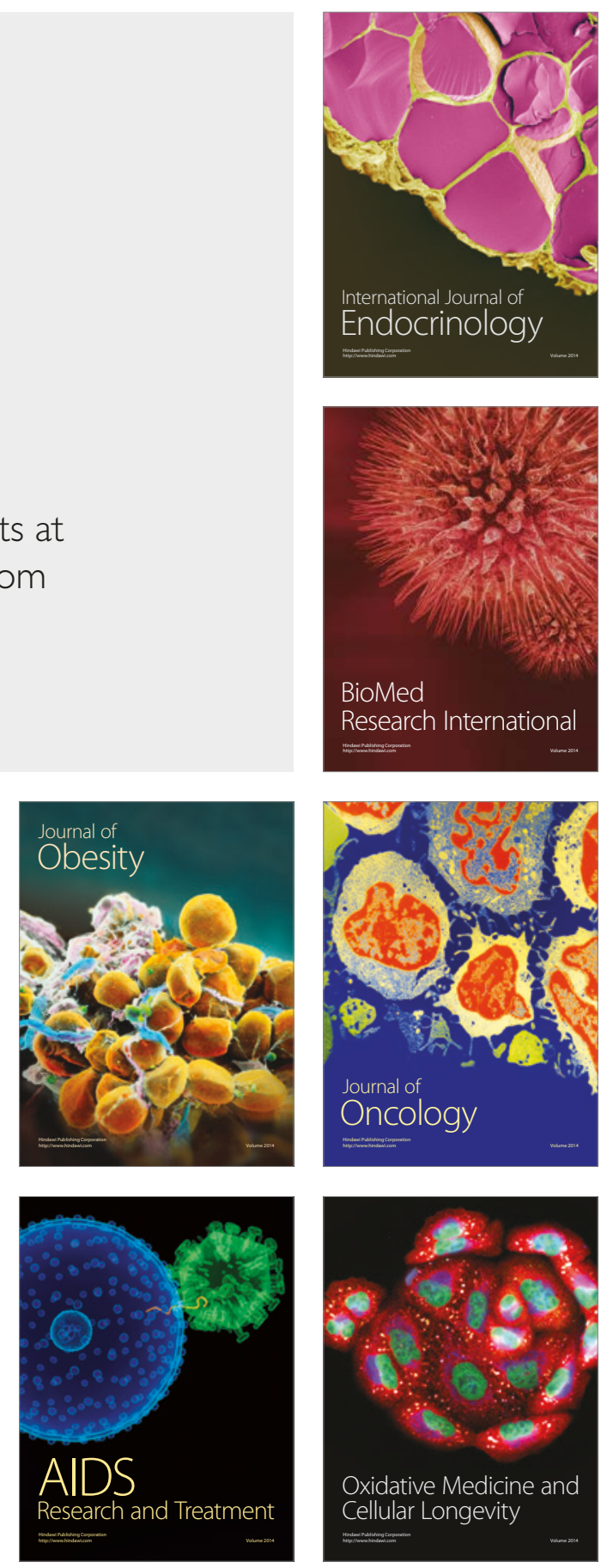